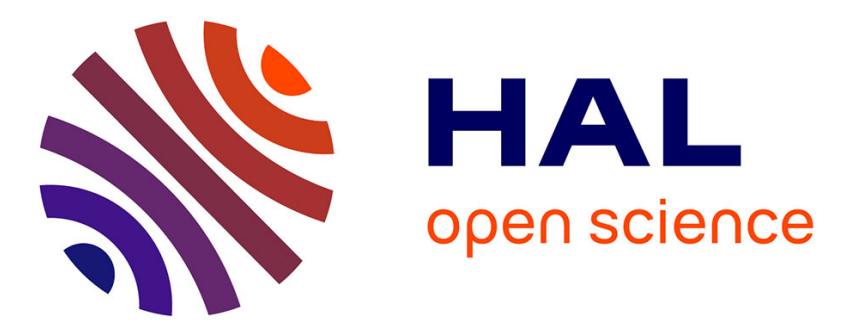

\title{
Présence du mythe unitaire bolivarien dans les traités d'alliance inter-latino-américains au XIXe siècle
}

\author{
Nathalie Blasco
}

\section{To cite this version:}

Nathalie Blasco. Présence du mythe unitaire bolivarien dans les traités d'alliance inter-latinoaméricains au XIXe siècle. Caravelle. Cahiers du monde hispanique et luso-brésilien, 2005, 85 (1), pp.185-204. 10.3406/carav.2005.2909 . hal-02133572

\section{HAL Id: hal-02133572 \\ https://hal.science/hal-02133572}

Submitted on 18 May 2019

HAL is a multi-disciplinary open access archive for the deposit and dissemination of scientific research documents, whether they are published or not. The documents may come from teaching and research institutions in France or abroad, or from public or private research centers.
L'archive ouverte pluridisciplinaire HAL, est destinée au dépôt et à la diffusion de documents scientifiques de niveau recherche, publiés ou non, émanant des établissements d'enseignement et de recherche français ou étrangers, des laboratoires publics ou privés. 
Présence du mythe unitaire bolivarien dans les traités d'alliance inter-latino-américains au XIXe siècle

Nathalie Blasco

\section{Citer ce document / Cite this document :}

Blasco Nathalie. Présence du mythe unitaire bolivarien dans les traités d'alliance inter-latino-américains au XIXe siècle. In: Caravelle, $n^{\circ} 85,2005$. Grandes plantations d'Amérique latine. pp. 185-204;

doi : 10.3406/carav.2005.2909

http://www.persee.fr/doc/carav_1147-6753_2005_num_85_1_2909

Document généré le 01/06/2016 


\section{Resumen}

RESUMEN- El modelo globalizador bolivariano se basa en la certidumbre de que una geopolítica de bloques era necesaria en el siglo XIX en el continente americano. A partir del Congreso Anfictiónico de Panamá (1826) todos los grandes proyectos unitarios latinoamericanos se concibieron según esta óptica : federaciones, confederaciones, alianzas permanentes. Se podía notar en la realización de asambleas, congresos, conferencias, tratados de amistad, los cuales, con grandes deficiencias, limitaciones, y pocos resultados, revelaban la existencia de una comunidad y un movimiento insistente hacia la unión.

\section{Résumé}

RÉSUMÉ- Le modèle bolivarien globalisant trouve son origine dans la certitude qu'une sorte de géopolitique de blocs était nécessaire au XIXe siècle sur le continent américain. A partir du Congrès Amphictyonique de Panama (1826) tous les grands projets unitaires latino-américains furent pensés dans cette perspective : fédérations, confédérations, alliances permanentes. On pouvait l'observer dans la tenue d'assemblées, de congrès, de conférences, de traités d'amitié qui, avec de grandes déficiences, des limites et peu de résultats, rendaient manifeste l'existence d'une communauté et d'un mouvement insistant vers l'union.

\section{Abstract}

ABSTRACT- The Bolivarian globalizing model has its origin in the strong belief that a kind of geopolitics based on blocks was necessary in the 19th century in the American continent. After Panama's Amphictyonic Congress (1826), all the great Latin American unitary projects were contemplated through this perspective : federations, confederations, permanent alliances. This can be observed in the way assemblies, congresses, conferences and friendship treaties were held, which, with great lacks, limits and few results, made obvious the existence of a community and of a movement aiming towards union. 


\title{
Présence du mythe unitaire bolivarien dans les traités d'alliance inter-latino-américains au XIXe siècle
}

PAR

\author{
Nathalie BLASCO \\ Université Michel de Montaigne - Bordeaux 3
}

Le XIXe siècle en Amérique latine fut une période d'intenses rapports internationaux : ce fut le siècle des traités. L'Argentin Mario Amadeo ${ }^{1}$ en dénombra plus de 10000 , qui furent conclus entre 1815 et 1914 , sur les thèmes les plus divers. Ces traités et ces accords, dont l'esprit n'avait que peu de rapport avec un quelconque unionisme, eurent pourtant des finalités affirmées : garantir le progrès et la prospérité du continent, opposer un front uni à l'ennemi extérieur commun (Espagne puis EtatsUnis principalement), puis stimuler l'échange économique souscontinental et les infrastructures de communication entre les pays latinoaméricains. Dans l'optique de déceler la présence du mythe de l'unité latino-américaine d'essence bolivarienne dans les relations inter-latinoaméricaines au XIXe siècle, il convient d'examiner la teneur des principaux traités d'alliance et de confédération signés au cours de cette période, car nombreux sont ceux qui révèlent une volonté d'évoluer en commun vers l'affermissement de l'indépendance politique.

En 1822 et 1823 , le gouvernement de Colombie signa des traités d'union, de ligue et de confédération perpétuelle avec le Pérou (6 juillet 1822), le Chili (21 octobre 1822), Buenos Aires (8 mars 1823), et le Mexique (3 octobre 1823), se plaçant à la tête des pays partisans de l'union. Le 7 décembre 1824, Bolívar, en tant que chef d'Etat du Pérou, invita les gouvernements indépendants du Guatemala (Amérique centrale), du Chili, du Río de la Plata (Argentine), du Mexique et de la Colombie (Grande) à envoyer des délégués à Panama en vue de la

1 M. Amadeo, Por una convivencia internacional. Bases para una Comunidad Hispánica de Naciones. Madrid, Ed. Cultura Hispánica, 1956. 
réunion d'un congrès général. Bolívar espérait donner suite aux alliances conclues par son pays et qui se présentaient comme de nécessaires préparations à la ratification d'une entente plus large.

\section{Les missions préparatoires au Congrès de Panama}

Pour Bolívar, l'union hispano-américaine devait être simultanée à l'indépendance, car il semblait impossible de la consolider sans réaliser l'union au préalable. Son intention, exprimée dans sa Lettre de la Jamaïque en 1815, était de réussir l'union sous forme de confédération entre les républiques hispano-américaines. Quand en 1819 fut constituée la Confédération grand-colombienne, Bolívar considéra qu'il avait consolidé l'axe autour duquel il pouvait proposer la tenue d'un congrès général. Il envoya deux commissions, l'une au Nord, l'autre au Sud, qui devaient engager formellement les gouvernements à collaborer et à participer activement à l'entreprise. Miguel de Santamaría fut envoyé vers le Mexique, et Joaquín Mosquera y Arboleda vers le Pérou, le Chili et Buenos Aires. Tous deux devaient obtenir la signature de traités bilatéraux, et en même temps réussir à obtenir l'adhésion d'autres gouvernements, de manière à ce que ces traités devinssent un ensemble d'accords qui, par multiplication, constitueraient un réseau d'accords multilatéraux.

Santamaría remplit sa mission avec succès : le Mexique et la Colombie signèrent un Traité d'Amitié, Ligue et Confédération le 3 octobre 1823 (Santamaría-Álamo). Dans le préambule du traité on pouvait lire la volonté "que cette ligue soit générale entre tous les Etats d'Amérique, pour que ceux-ci, unis, forts et puissants, puissent soutenir ensemble la cause de leur indépendance 2 ". Les deux nations se déclaraient " unies, liées et confédérées désormais et pour l'avenir, en paix et en guerre", dans le but de réunir leurs efforts contre l'Espagne et toute autre puissance étrangère, et de "garantir après leur reconnaissance, leur prospérité mutuelle, la plus grande harmonie et leur bonne intelligence ". Les objectifs du traité allaient plus loin que la simple consolidation de l'indépendance, car les signataires se promettaient une union permanente et s'engageaient à se concerter pour leurs relations avec les nations étrangères.

Quant à Mosquera, il conclut facilement un Traité d'Union, Ligue et Confédération avec le Pérou, qui fut signé à Lima le 6 juillet 1822. Son contenu était similaire à celui du traité souscrit avec le Mexique : il stipulait la défense mutuelle, l'égalité de droit des citoyens à l'intérieur des deux nations, la libre importation et exportation réciproque, et il

2 Cité par Edmundo A. Heredia, "Primeras misiones integracionistas latinoamericanas (1810-26) ", Anuario de Estudios Hispanoamericanos. Sevilla, L, n², 1993, p. 220. 
invitait à une Assemblée Générale d'Etats Américains qui devait se réunir à Panama. Mosquera atteignit également son deuxième objectif puisqu'il parvint à signer un Traité d'Union, Ligue et Confédération avec le Chili, dont le contenu était identique au précédent. Sa démarche fut alors aidée par un allié de valeur, Bernardo O'Higgins, fervent admirateur de Bolívar.

Mais à Buenos Aires l'itinéraire de Mosquera se trouva bloqué, car il dut faire face à la position intransigeante de Rivadavia qui refusait tout engagement avec les nouvelles républiques. Le ministre argentin trouvait nécessaire de négocier séparément avec l'Espagne ou une autre nation européenne pour la reconnaissance de l'indépendance. Il comptait davantage sur la Grande-Bretagne. Les deux hommes s'en tinrent à un Traité d'Amitié et d'Alliance, le 8 mars 1823, qui engageait les deux gouvernements de Colombie et de Buenos Aires.

Ces traités bilatéraux signés par la Colombie avec des "pays frères" se voulaient l'amorce du traité multilatéral de confédération auquel voulait aboutir Bolívar. Le 7 décembre 1824, depuis Lima, deux jours avant la victoire gagnée à Ayacucho, Bolívar, en tant que Président du Pérou, pressentant la fin de la domination espagnole, expédia la circulaire d'invitation au Congrès de Panama. L'objectif fondamental de cette réunion était d'obtenir le système de garanties qui, en état de guerre ou de paix, fût le bouclier du destin des républiques hispano-américaines. Le chef d'Etat du Pérou ne souhaitait pas la mise en place d'une simple organisation offensive et défensive dictée par l'urgence, mais la constitution d'une entité juridique qui permît la solution pacifique des conflits entre pays hispano-américains et qui fût apte à défendre leurs intérêts.

\section{Le Congrès Amphictyonique de Panama}

\section{Un congrès de " tous " les Etats hispano-américains?}

La circulaire fut adressée aux gouvernements de Colombie, du Mexique, du Río de la Plata, du Chili et d'Amérique centrale. Cependant, en l'absence du Libertador, les gouvernements de Colombie, du Mexique et d'Amérique centrale prirent l'initiative d'inviter les EtatsUnis à y participer. La correspondance privée de Bolívar montre bien, cependant, qu'il excluait les Etats-Unis de la réunion qu'il projetait. Il les voyait comme des étrangers, pas seulement en raison de leur origine, de leur langue, de leur religion et de leurs coutumes, mais surtout en raison de la position neutre qu'ils avaient adoptée face aux luttes pour l'indépendance hispano-américaine. Finalement, furent invités en tant qu'observateurs les Etats-Unis, la Grande-Bretagne, la France et les PaysBas, mais les délégués nord-américains n'arrivèrent jamais. Quant au Río 
de la Plata et au Chili, les deux pays se montraient jaloux et méfiants face à Bolívar et à ses ambitions; par conséquent ils n'envoyèrent aucun délégué.

La participation du Brésil fut problématique : d'une part, il était un acteur majeur de l'Amérique émancipée, mais d'autre part, son régime monarchique était inconciliable avec les bases du congrès général projeté. Bolívar l'excluait de son projet d'union car il voyait en lui une sorte d'agent de la Sainte Alliance européenne dont la politique était toujours opposée à l'établissement de toute république hispano-américaine. Les Provinces Unies du Río de la Plata se méfiaient du danger que représentait la volonté brésilienne de s'emparer de l'Uruguay, et le gouvernement brésilien n'obtint aucune crédibilité quand il proclama sa décision d'intégrer le système américain et d'encourager des projets fédératifs. Le désir de leadership du Brésil dans une éventuelle confédération ou ligue avait été exposé de manière répétée par ses ministres et ses diplomates. Cette ambition ne pouvait être acceptée par les républiques hispano-américaines, car elles y voyaient la forme explicite de la volonté brésilienne de se placer à la tête de ces pays, avec la menace conséquente d'hégémonie et d'expansion.

Enfin, deux Etats furent délibérément " oubliés " : jamais le Paraguay ne fut considéré comme possible invité (le dictateur Gaspar Rodríguez de Francia maintenait, depuis 1816 , le pays dans un isolement autoritaire), et Haïti fut également laissé de côté, en raison de l'origine africaine de sa population et afin d'éviter l'opposition de la France.

Le "Congrès Général " ne compta donc que quatre participants (le Mexique, l'Amérique centrale, le Pérou et la Colombie), une situation qui tendait à prouver le manque d'ambition politique généralisé des républiques hispano-américaines et le caractère idéaliste du projet d'une entente continentale aussi structurée pour l'époque.

\section{Le grand défi lancé par le Congrès de Panama}

La finalité du congrès convoqué par Bolívar était de démontrer la capacité de réussir l'union et d'exhiber une force suffisante pour défendre sous une forme conjointe les indépendances et les libertés gagnées par la révolution.

La Colombie et le Mexique formulaient des propos particulièrement définis. La Colombie devait rénover le traité d'union, ligue et confédération perpétuelle contre l'Espagne ou n'importe quelle autre puissance qui pourrait tenter de la dominer, considérer la libération de Cuba et Porto Rico, conclure des accords commerciaux et consulaires, examiner les moyens pour donner effer aux principes énoncés par le Président Monroe, et établir les principes de Droit International Public Américain. Quant au Mexique, il souhaitait la garantie commune de 
l'indépendance politique et de l'intégrité territoriale, soutenir des formes républicaines de gouvernement, rejeter la colonisation par des nations étrangères, et fixer les principes généraux sur lesquels devait reposer le Droit Public Américain.

Du 22 juin au 15 juillet 1826 eurent lieu les sessions du Congrès Amphictyonique de Panama3. Les quatre gouvernements participants signèrent un Traité d'Union, Ligue et Confédération perpétuelle ${ }^{4}$, puis un accord sur la défense commune, ainsi qu'un engagement à se réunir postérieurement à Tacubaya, au Mexique, où les traités devaient être ratifiés. Le propos du Traité Perpétuel était de protéger la souveraineté et l'indépendance de chacun des pays signataires contre toute tentative, de la part des puissances étrangères, d'intervention sur les territoires de la Confédération. Cela signifiait une alliance défensive, et la première tentative moderne d'introduire les concepts de sécurité collective et d'arbitrage, car en cas de conflits entre les pays, l'Assemblée Générale de la Ligue devait les résoudre.

Le Traité stipulait la paix indivisible, aucune des parties ne pouvant faire la paix avec des ennemis communs sans $y$ inclure tous les autres alliés. Afin d'éviter une politique belliqueuse et dangereuse pour les intérêts collectifs, aucune des parties ne pouvait conclure de traités d'alliance avec une quelconque puissance extérieure sans consulter auparavant la Confédération. De plus, pour régler pacifiquement les conflits, les pays avaient l'obligation de porter leurs différends devant l'Assemblée pour y être réglés sous forme conciliatoire. Les parties s'engageaient en outre à coopérer à la complète abolition du trafic d'esclaves d'Afrique, considéré comme un crime de piraterie. Les citoyens des pays signataires pouvaient également exercer leur profession dans un quelconque de ces pays sans distinction. Enfin, pour ne pas déshonorer la Confédération, il existait des clauses pénales avec exclusion temporaire, tant pour ceux qui oublieraient les procédés pacifiques que pour ceux qui transformeraient leur condition républicaine en régime monarchique. La souveraineté et la participation à un schéma international n'étaient pas considérées comme incompatibles, le droit international étant inconcevable sans souveraineté des Etats.

Malgré tous ces engagements, seule la Colombie ratifia le traité. L'assemblée manqua d'appui politique. Son travail lors de la Conférence de Tacubaya en 1828 se limita donc aux domaines juridiques et académiques. Les convulsions politiques de l'Amérique hispanique étaient manifestes : le Mexique connaissait une insurrection, l'Amérique

3 Documentation complète relative au congrès dans : El Congreso de Panamá (1826), recop. y pról. por Raúl Porras Barrenechea, Lima, imp. "La Opinión Nacional ", 1930, Cl-500 p. (Archivo diplomático del Perú, $n^{\circ} 1$ ).

${ }^{4}$ Le texte complet du traité est notamment reproduit dans un ouvrage plus récent: Vargas, Francisco Alejandro, El Congreso anfictiónico de Panamá y su proyección hacia el futuro de la América, Caracas, Oficina Técnica M. D., 1976, p. 111-120. 
centrale était en guerre civile, le Pérou et la Colombie s'affrontaient, cette dernière était en voie de dissolution, bref, un chaos généralisé régnait dans les années immédiatement postérieures à l'Indépendance, conduisant à l'échec de la Confédération. Le 9 octobre eut lieu la dernière Conférence de Tacubaya au Mexique entre plénipotentiaires du Mexique, d'Amérique centrale et de Colombie : tout resta à l'état de projet, et ainsi se dilua le rêve bolivarien.

\section{L'échec du congrès}

Les causes de l'échec du Congrès de Panama furent variées et révélèrent sans doute le manque de préparation des dirigeants hispanoaméricains de l'époque à appréhender les questions internationales. D'une part, l'instabilité politique des gouvernements ne permit pas de mettre en pratique les aspirations formulées, d'autre part, il existait un manque d'intérêt réciproque entre les peuples (accentué par des difficultés de communication et une dépendance chronique des excolonies face à l'Espagne), ainsi qu'un manque de vision chez les hommes d'Etat pour traiter en commun les problèmes hispano-américains. Mais les causes profondes de l'échec du Congrès vintent de l'opposition manifestée par plusieurs pays. Les Etats-Unis, qui avaient toujours maintenu une position de neutralité par rapport aux guerres d'indépendance hispano-américaines et qui, suite à la formulation de la Doctrine Monroe (1823), élaboraient leur politique extérieure en fonction de leurs intérêts propres, ne se montraient pas prêts à accepter un accord global quelconque. Ce pays était de plus persuadé, avec la Grande-Bretagne, que le congrès était purement diplomatique et qu'il n'avait aucune capacité législative. Mais les deux puissances rejetaient surtout l'entente entre la Colombie et le Mexique qui envisageaient d'agir ensemble et par la force en faveur de l'indépendance de Cuba et de Porto Rico.

De leur côté, le Chili et l'Argentine avaient décidé d'une absence de leurs représentants au congrès. Ces deux pays manifestaient ainsi le sentiment d'impopularité que leur inspirait Bolívar lui-même, identifié au projet par l'initiative de sa convocation. La Constitution de la Bolivie, fraîchement instituée par le Libertador, attribuait des pouvoirs étendus au président, et semblait par là même, aux yeux de ces Etats du Sud, annoncer une ambition continentale et des prétentions à un pouvoir suprême. Ces derniers craignaient une autocratie de la part de Bolívar, et donc un éventuel étouffement des fédérations et des autonomies nationales récemment acquises. Le gouvernement argentin de Rivadavia en particulier avait, de toute façon, une opinion contraire à celle de Bolívar dans la mesure où il ne craignait pas l'agression européenne. Sa participation aurait eu lieu sans intention de s'engager dans des causes 
étrangères à son intérêt propre. L'Argentine de Rivadavia affirmait ses institutions politiques séparément du contexte hispano-américain et se détachait ostensiblement de la problématique générale du sous-continent. Sa préoccupation était plutôt d'obtenir le respect des puissances européennes et de revendiquer, à cette fin, une singularité par rapport au reste du continent. Il convient également de souligner l'absence de l'instigateur du projet : Bolívar lui-même ne s'est pas rendu à Panama. Comment était-il possible, alors, de lancer un projet aussi grand si le plus grand n'en prenait pas la tête? L'autorité supérieure du Libertador, acquise par le prestige politique et par la victoire sur les champs de bataille, était l'appui nécessaire à l'union désirée.

Les positions isolationnistes se confirmant et les unionistes ayant reculé, les nations naissantes célébrèrent comme conquête maximale l'indépendance politique propre. Elles durent dissimuler et justifier l'échec de la réunion et de la Confédération, argumentant que ces tentatives avaient été la négation des souverainetés, quand en définitive elles étaient la forme la plus appropriée aux circonstances pour les confirmer. Il était manifeste que l'Amérique hispanique marchait dans le sens inverse à celui que lui montrait Bolívar: elle allait vers le morcellement territorial et les luttes intestines. Cependant, en marge de cette désagrégation se développait un mouvement insistant vers la connexion de ces pays fraîchement livrés à la vie indépendante. On pouvait l'observer dans la tenue d'assemblées, de congrès, de conférences, de traités d'amitié qui, avec de grandes déficiences, des limites, et peu de résultats, rendaient manifeste l'existence d'une communauté sans pacte écrit. Car, malgré l'échec du Congrès de Panama, Bolívar lança à cette occasion une multitude d'idées qui rappelaient la Grèce Antique (notamment l'idée de confédération de nations libres, la notion de sécurité collective, le concept d'arbitrage international pour la solution pacifique des conflits) et qui, comme une semence, allaient germer plus tard. Si le congrès de 1826 est resté célèbre, c'est qu'il constitua le point de départ d'un processus pour lequel il reste une référence aujourd'hui encore, et qu'il a stimulé, de plus, l'idée unioniste qui n'a jamais cessé d'exister dans l'esprit des dirigeants et des intellectuels latino-américains.

\section{Le modèle globalisant et fédéral bolivarien}

L'idée d'un congrès général et permanent signifiait que celui-ci devait être l'organe principal de la Confédération. Ce congrès de ministres plénipotentiaires devait réunir les caractéristiques d'un pouvoir exécutif et législatif et d'un pouvoir judiciaire. Il devait résoudre les problèmes de limites frontalières et disposer du contingent militaire de chaque Etat. Pour Bolívar, la construction d'un édifice politique véritablement fédéral dépendait de l'adoption de lois supranationales, d'efforts autour d'un 
idéal commun, de la permanence et de la périodicité du congrès. Tel était le modèle de sa "nation de Républiques". Le modèle bolivarien globalisant trouvait son origine dans la certitude qu'une sorte de géopolitique de blocs était nécessaire au XIXe siècle sur le continent américain, mais en s'appuyant sur une idée culturelle. La notion d'américanisme "méridional ", affirmation culturelle et indépendantiste vis-à-vis de l'Espagne, servait de base à l'idéologie politique fédéraliste de Bolívar. Mais ce modèle avait été conçu lors des étapes initiales de formation des Etats hispano-américains indépendants, d'où la difficulté pour les dirigeants nationaux en place (Rivadavia, Francia...) de penser, déjà, une extension de l'Etat national, voire une communauté continentale. Tous les grands projets unitaires furent pensés au début du XIXe siècle dans cetre perspective, et furent formulés par quelques groupes dirigeants des nouveaux pays poussés par l'idéalisme de la geste émancipatrice : on parlait de fédérations, de confédérations ou d'alliances permanentes. Cette orientation se manifesta à travers les premiers liens crés par et entre les Etats, et surtout à travers les congrès régionaux, à partir de 1826. Il s'agissait de promouvoir la fusion politique de la région grâce à un rapprochement graduel et formel qui impliquait des pactes défensifs et l'adoption de principes généraux, ainsi que des instruments spécifiques qui consacraient la solidarité et l'indépendance politique des Etats hispano-américains.

\section{Les « congrès d'alliances "}

L'échec des grands projets unitaires ne fut pas un obstacle au développement de divers schémas d'alliance défensive et offensive pendant le XIXe siècle, qui tendaient à reproduire les systèmes d'équilibre de pouvoir qui avaient cours en Europe à la même époque (la Sainte Alliance par exemple). Il s'agissait de mécanismes beaucoup plus flexibles et informels, centrés sur une perception différente des intérêts nationaux selon les Etats, mais qui, de ce fait, contenaient plus d'éléments de conflit que de coopération. Examinons les congrès les plus marquants, et qui suivirent chronologiquement le Congrès de Panama.

\section{Le Congrès Américain de Lima : 1847-48}

Cette réunion fut convoquée face à la menace (qui ne se concrétisa pas) d'une intervention contre l'Equateur commanditée par l'Espagne avec la complicité de la Grande-Bretagne. On craignait une extension au reste du continent. Lors du congrès réuni à Lima du 11 décembre 1847 au $1^{\text {er }}$ mars 1848, les participants (Pérou, Bolivie, Chili, Equateur et Nouvelle-Grenade) évoquèrent, tout comme lors de la première réunion 
américaine de Panama, l'oppression subie pendant la colonie et la fragilité de leur indépendance et de leurs institutions. Les pays participants mirent sur pied un Traité de "Confédération Américaine" qui stipulait la défense de l'intégrité territoriale et l'indépendance politique des Etats confédérés, la création d'une alliance défensive contre toute agression extérieure, l'unification du droit des gens américain, la fixation des frontières des nouvelles républiques, la défense des démocraties, l'abolition de l'esclavage, et la nécessité de résoudre pacifiquement les conflits. Un traité de commerce, un accord postal et un accord consulaire furent également signés. Enfin, les participants insistèrent sur leur communauté d'origine, de langue, de religion, de coutumes, une position géographique particulière et une réciprocité d'intérêts; ils tentaient ainsi de s'affirmer à travers la revendication d'une communauté de culture et à travers l'image d'une même et unique nation.

Les références au traité d'Union, Ligue et Confédération de 1826 étaient manifestes; on retrouvait, entre autres, la volonté de s'assurer d'une action commune en cas de danger (extérieur surtout) et de renouveler les possibilités d'une entente perpétuelle. De plus, l'arbitrage international, la défense de l'intégrité territoriale, l'indépendance politique, la création d'une alliance défensive et d'autres propositions à caractère législatif étaient mentionnés tout comme en 1826, ce qui révèle plus qu'une simple similitude des stipulations des deux traités et une réelle intention de tenter à nouveau l'expérience fondatrice avortée. Ce traité ne fut pourtant pas ratifié, des divergences survenant à propos de certains problèmes secondaires ou du fonctionnement même de la confédération projetée.

Cependant, l'invasion annoncée de l'Equateur n'avait finalement pas eu lieu, mais au mois de mai 1846 , ce fut le Mexique qui subit l'invasion des Etats-Unis. Quand le Congrès Américain de Lima se termina, cette invasion avait déjà été entérinée par le Traité Guadalupe Hidalgo en février 1848, par lequel à l'annexion antérieure du Texas succédait la perte définitive de l'Arizona, de la Californie, du Nevada, du NouveauMexique et de l'Utah. Malgré cet événement fondamental pour l'avenir de l'ensemble des républiques hispano-américaines, l'invasion nordaméricaine, qui avait évolué en conquête, ne suscita aucune réaction. Une fois le danger espagnol écarté, les Etats hispano-américains avaient sous-estimé le danger nord-américain et avaient surtout montré qu'une conscience sous-continentale réelle leur faisait défaut. Ils n'étaient pas encore capables de percevoir le nouveau danger qui s'additionnait au danger européen toujours présent, un danger qui désormais provenait de leur propre hémisphère 5 .

5 Pour plus de détails sur ce congrès, voir : Arosemena, Jusro, "Estudio sobre la Idea de una Liga Americana ", Tareas, Panamá, n²6, jun-nov. 1973, p. 61-64. 


\section{Le Traité " tripartite" de 1856}

En 1855 et 56 , des événements se produisirent en Amérique centrale qui ravivèrent l'idée d'union. A cette époque, la menace d'une intervention de l'Europe contre l'Amérique hispanique, et qui avait jusqu'alors inspiré les projets d'union, sembla ne plus exister. En revanche, les expéditions réitérées du flibustier William Walker en Amérique centrale provoquèrent un sentiment d'hostilité face aux EtatsUnis qui devinrent le nouvel ennemi de l'Amérique méridionale. C'est cette menace qui motiva la réunion du 15 septembre 1856.

Ce jour-là, à Santiago du Chili, le Pérou, l'Equateur et le Chili conclurent et signèrent un traité appelé "continental " par certains et "tripartite" par d'autres, qui se proposait de créer les bases d'une "Union pour les Républiques Américaines" (sans les Etats-Unis mais avec le Brésil). Ce traité exposait des principes généraux sur le Droit Public International Américain, interdisait les expéditions agressives contre un Etat de la Ligue, qu'elles proviennent ou non de l'un des Etats de l'Union, déclarait nécessaires des démarches conciliatoires préalables avant d'entrer en conflit et projetait la création d'un congrès de plénipotentiaires. Fut également établi le droit de chaque pays à conserver son autonomie et l'intégrité de son territoire, et il fut concédé à chaque peuple concerné par le traité le droit de jouir sur le territoire des autres des mêmes droits que les nationaux.

Les pays signataires accordèrent une importance plus grande à l'adoption de certains principes internationaux qu'à la formation d'une ligue proprement dite. Il n'y avait dans l'union imaginée par le traité tripartite aucune action commune, quelle qu'elle fût, sauf pour rejeter les expéditions des flibustiers; une telle union n'était que nominale. Mais au moment de ratifier le traité, chaque pays ajouta ses propres modifications, rendant impossible la ratification. De plus, l'absence de dispositions exclusivement communes renforça l'échec de l'initiative continentale6.

Néanmoins, à la même époque, les représentants de plusieurs Etats hispano-américains se réunissaient à Washington: le Mexique, le Guatemala, le Salvador, le Costa Rica, la Nouvelle-Grenade, le Venezuela et le Pérou signaient le 9 novembre 1856 un "Projet d'Alliance" défensive régionale centraméricaine, qui d'une part rejetait les invasions de Walker et d'autre part établissait les bases de la "Confédération des Etats Hispano-américains ". Il semblait alors qu'en dehors de leurs frontières (la réunion eut lieu à Washington), les représentants hispanoaméricains avaient plus de facilité à envisager la communauté d'éléments qui les unissaient.

6 Pour plus de détails sur les discussions et les clauses des traités de la Conférence de Santiago : ibid., p. 64-73. 
Cette remarque s'appuie également sur la naissance, la même année (1856), à Paris et parmi les exilés latino-américains, d'une nouvelle conscience. Les agressions des Etats-Unis sur le Mexique, qui s'étendaient vers l'isthme centraméricain, semblaient peu à peu devenir continentales. C'était tout naturellement que l'opposition Etats-Unis/Mexique se transformait en une opposition Amérique du "Nord"/ Amérique du "Sud "7. C'est-à-dire qu'au traditionnel antagonisme Europe/Amérique qui conditionnait l'unionisme hispano-américain depuis l'Indépendance succédait un autre antagonisme, interne à l'hémisphère, qui opposait deux Amériques. Ce fut le Colombien José María Torres Caicedo qui exprima le premier la prise de conscience de ce vaste antagonisme intrahémisphérique, en opposant dans son poème Les Deux Amériques la " race saxonne" à la " race latine " 8 . C'est d'ailleurs dans cet écrit qu'il formula le nom "Amérique latine", une dénomination à caractère militant et profondément unioniste.

\section{Le deuxième Congrès Américain de Lima : 1864-65}

Comme ce fut toujours le cas au XIXe siècle, c'est la crainte d'un danger venant de l'extérieur qui fut à l'origine de la réunion des délégués de certains pays latino-américains en 1864-1865 à Lima. D'une part, l'Espagne reconquit Saint-Domingue en 1861 et tenta de reconquérir le Pérou en 1864 en envahissant les Iles Chinchas, et d'autre part, la France livra une guerre de conquête au Mexique pour substituer un empire à une république (1862) et y imposer Maximilien. De plus, l'influence politique et commerciale des Etats-Unis sur les pays hispano-américains s'affirmait progressivement suite à l'annexion de certaines parties du Mexique et à l'invasion du Nicaragua.

Le Deuxième Congrès Américain de Lima, projeté par le Traité "tripartite" de 1856, se déroula donc dans un esprit prioritaire de résistance aux agressions étrangères. Le gouvernement péruvien envoya une convocation à laquelle répondirent favorablement la Bolivie, le Chili, l'Equateur, la Colombie, l'Argentine et le Venezuela. Les finalités de cette réunion étaient de défendre les institutions des républiques latinoaméricaines, de favoriser l'échec des tentatives de domination éventuelles et de faciliter les relations commerciales entre pays hispano-américains. Plusieurs accords furent signés : un Traité d'Union et d'Alliance défensive, un Traité de Maintien de la Paix, un Traité sur le courrier et un Traité de Commerce et de Navigation notamment. On reconnut la validité du principe des démocraties puis on dénonça la prépondérance du pouvoir nord-américain, tout comme son extraordinaire agressivité.

7 Considérons ces appellations en termes de géographie politique.

8 "Las Dos Américas ", 26 de septiembre de 1856, El Correo de Ultramar, Paris, 17 février 1857. 
Malgré l'objectif principal du congrès de confirmer l'idée d'une alliance régionale, le résultat fut décevant : certaines républiques participèrent au congrès mais déclarèrent leur neutralité dans la guerre opposant l'Espagne au Pérou (l'Argentine refusa de s'allier au bloc Pacifique), d'autres réclamèrent un traitement particulier (le Chili revendiqua sa situation exceptionnelle en raison de la particularité de ses frontières), et certains autres discutèrent la participation d'autres pays aux négociations (devaient-ils inclure les Etats-Unis et le Brésil ?). Le congrès révéla des divergences de points de vue, et il connut le même sort que les précédents pour avoir, entre autres, seulement exprimé les aspirations de quelques secteurs.

Les années 1860-1865 ne sont pourtant pas sans intérêt pour le processus de formulation d'un unionisme latino-américain : l'année 1862 marque le lancement au Chili, sous la présidence du Général Gregorio Las Heras, du mouvement intellectuel et politique unioniste Unión Americana, un mouvement d'opinion et d'association qui s'organisa dans les grandes capitales et qui eut une influence idéologique certaine durant une décennie. Quant à l'année 1865 , date du $2^{\circ}$ Congrès de Lima, elle célèbre la publication, à Paris, du livre de Torres Caicedo, Unión LatinoAmericand, véritable plaidoyer pour une union latino-américaine qui consacre les initiatives du précurseur incontestable Simón Bolívar.

\section{Le Congrès de Droit International Sud-américain de Montevideo : 25 août 1888 - 18 février 1889}

Après 23 ans passés sans la tenue d'une conférence contincntale latino-américaine, un congrès "sud-américain " fut organisé en 1888 suite au projet, annoncé par le Secrétaire d'Etat des Etats-Unis James G. Blaine en 1881, de réunir une conférence panaméricaine avant 1890. C'est-à-dire que ce congrès eut lieu au moment même où était lancé le Congrès de Washington de 1889-90 (la circulaire d'invitation est datée du 13 juillet 1888) pour tenter de prendre de court les Nord-américains. Il fallait éviter que ceux-ci n'imposent aux Etats latino-américains certaines mesures commerciales et politiques désavantageuses.

Le congrès avait pour objectif d'établir les bases des relations entre pays latino-américains sur une législation uniforme en matière de droit international privé. Dans cette optique, les participants (Argentine, Brésil, Bolivie, Chili, Paraguay, Pérou et Uruguay) ratifièrent huit traités et conventions : de droit civil international, de droit commercial international, de droit pénal international, de code de procédure, de marques de commerce et de fabrication, de brevets d'invention, et sur la

9 Torres Caicedo, J. M., Unión Latino-Americana, Paris, Rosa y Bouret, 1865. 
propriété littéraire et artistique, ainsi qu'une convention sur l'exercice des professions libérales.

La réunion de ce congrès avait trouvé son origine dans un défaut d'unité entre les législations des divers pays et avait pour but d'éliminer un esprit particulariste. Le résultat de ce congrès fut véritablement positif puisque les traités signés furent également ratifiés et que les signataires étaient des gouvernements exclusivement hispano-américains. On peut le définir comme foyer réel d'unification, et d'autant plus quand on sait que les délégués argentins étaient les mêmes qui se rendirent à Washington pour la première conférence panaméricaine, et qu'ils s'opposèrent aux projets panaméricanistes.

\section{Le Congrès centraméricain de San Salvador : 15 octobre 1889}

Cette réunion entre les cinq pays de l'isthme centraméricain fut la dernière d'une série qui commença en septembre 1885, quand le Guatemala, le Salvador et le Honduras signèrent le Traité Ramírez-MezaZelaya qui établissait les premières bases pratiques de reconstitution de l'ancienne union centraméricaine (1823-1838). Les causes de cette renaissance semblaient provenir de la bataille que se livraient, depuis le milieu du siècle, la Grande-Bretagne et les Etats-Unis pour affirmer leur influence dans la zone centraméricaine. Dès 1850, le Traité ClaytonBulwer avait défini le partage d'influences entre les deux pays dans la région de l'isthme. Mais à la fin du XIXe siècle, les Etats-Unis prétendaient en finir avec la suprématie britannique sur le continent latino-américain, et s'intéressaient déjà à l'acquisition des droits français sur la zone de construction du canal interocéanique.

En 1889 à San Salvador fut signé le "Pacte d'union provisoire des Etats d'Amérique Centrale ". Inauguré le 15 septembre 1890, le pacte établissait un pouvoir exécutif fédéral, laissait à chaque Etat la pleine autonomie dans ses affaires intérieures et concluait les négociations centraméricaines qui depuis 1885 prévoyaient la reconstruction de l'union centraméricaine. Les dirigeants centraméricains étaient alors motivés par les concepts politiques de Bolívar et l'action unificatrice de Morazán. Ils reprenaient leurs idées fondatrices dans le microcosme de l'isthme, mus par une hostilité et une crainte envers les poussées hégémoniques nord-américaines. Grâce à la signature du pacte de San Salvador, considéré comme un pacte d'union provisoire devant conduire à l'union définitive, la volonté unioniste se concrétisa en une union régionale, la Fédération centraméricaine, et bien que celle-ci n'ait pas duré, elle constitua une étape supplémentaire vers le rapprochement continental latino-américain fondé sur une communauté de culture et une même nécessité stratégique et politique. 
La multiplication des réunions était due essentiellement à la peur du danger extérieur, notamment face aux tentatives de reconquête espagnole de certains territoires, et face à l'expansion des Etats-Unis à plusieurs niveaux : territorial, économique, et politique. Lors des réunions qui se tinrent épisodiquement au XIXe siècle en Amérique hispanique furent formulées des lois transcendantes pour le continent : la nécessité de l'arbitrage international, la défense de l'intégrité territoriale des Etats, le droit à l'indépendance politique, l'unification du droit des gens américain, la reconnaissance du principe républicain, l'existence d'une région centraméricaine "fédéralisable", le devoir de méfiance envers les Etats non latino-américains intéressés par des relations internationales avec les pays d'Amérique latine. C'est aussi lors des congrès du XIXe siècle que naquit la coutume de l'association et de la concertation.

Cependant, l'interaction des mécanismes et des schémas issus de ces réunions dessina un système latino-américain relativement instable, marqué par des conflits et des guerres (prise de Mexico par le gouvernement de Polk en 1847, invasion du Nicaragua par W. Walker en 1856, conquête de Saint-Domingue par les troupes espagnoles en 1861 , intervention française au Mexique à partir de 1862, intervention espagnole sur les Iles Chinchas en 1863, Guerre de la Triple Alliance entre 1865 et 1870 , Guerre du Pacifique entre 1879 et 1883), et par des séquelles de rivalités et de tensions (frontières longtemps contestées entre le Chili, le Pérou et la Bolivie, entre le Chili et l'Argentine, ou entre le Brésil et l'Argentine, par exemple) qui persistèrent jusqu'au XXe siècle, et dont profita sans retenue l'idéologie panaméricaniste qui pointait.

\section{La Conférence internationale américaine de Washington : $1889-90$}

\section{Le Congrès de Washington : 2 octobre 1889 - 19 avril 1890}

On doit l'initiative de la réunion de ce congrès panaméricain au Nord-américain James G. Blaine, secrétaire d'Etat sous la présidence de Garfield. Il invita les républiques américaines à se réunir à Washington, officiellement pour rechercher ensemble des principes d'arbitrage et des solutions de paix aux conflits frontaliers réitérés, et plus particulièrement à celui qui avait cours : la Guerre du Pacifique (qui opposait le Chili au Pérou et à la Bolivie). Cependant cette première initiative ne put avoir lieu en raison de la démission de Blaine suite à l'assassinat du Président Garfield. Mais lorsque Blaine revint au Secrétariat d'Etat en 1888 sous la présidence de Harrison, celui-ci réaffirma immédiatement ses intentions, donnant suite à l'invitation lancée par son prédécesseur Bayard aux Républiques du Mexique, de l'Amérique centrale et du Sud, de Haïti, 
Saint-Domingue et du Brésil afin qu'elles se réunissent à Washington en 1889.

L'objectif de Blaine était de créer une union douanière et monétaire entre l'Ámérique du Nord, centrale et du Sud. Mais rapidement l'objectif nord-américain inavoué de mainmise sur l'économie latino-américaine fut découvert. En réalité, le projet de Blaine se résumait à ceci : un échange de produits manufacturés des Etats-Unis contre des matières premières d'Amérique latine. Ce qui signifiait que les Etats-Unis, qui ne trouvaient pas encore de concurrence manufacturière sérieuse de la part des Latino-américains eux-mêmes (mais souhaitaient déloger l'Europe, et la Grande-Bretagne surtout), auraient trouvé un continent entier ouvert à la vente de leurs produits manufacturés, alors que l'Amérique latine, en fermant les portes de son commerce d'exportation à l'Europe par son adhésion au Zollverein américain, n'aurait plus eu d'autre solution pour sa production agricole et ses matières premières que celle des Etats-Unis, eux-mêmes déjà abondamment fournis en ressources naturelles. Il s'agissait donc pour les Latino-américains d'aliéner leur liberté commerciale aux Etats-Unis par des traités de réciprocité exclusive favorables à ces derniers, et d'exclure et d'isoler l'Europe. C'était l'idée panaméricaniste qui s'affirmait alors, apportant une extension dans le domaine économique à la Doctrine Monroe : l'union hémisphérique sous l'hégémonie des Etats-Unis.

L'initiative fut mal accueillie par les Etats latino-américains qui ne voyaient pas la nécessité d'une tutelle de l'union nord-américaine pour maintenir leurs relations économiques et la garantie de leurs droits souverains. Ils ne reconnaissaient pas l'ennemi hypothétique que leur montraient les Etats-Unis (l'Europe) et ne voulaient pas s'associer au plan d'exclusion et d'isolement dirigé par les Etats-Unis contre le Vieux Continent.

L'autre temps fort de la conférence fut le débat concernant un projet d'arbitrage. Le projet de traité qui fut présenté avait été établi sous l'autorité de l'Argentin Quintana : celui-ci proposait un arbitrage obligatoire pour toutes les disputes qui auraient lieu entre Etats américains, mais le projet ne répondait pas au souhait des Etats-Unis qui pensaient obtenir l'autorité de l'arbitrage continental, avec un tribunal permanent siégeant à Washington. Et même si, par la soumission au vote d'un tel projet, l'on pouvait voir " la victoire indiscutable et totale de la pensée hispano-américaine concernant l'arbitrage, nettement opposée à la pensée des Etats-Unis 10 ", une fois les positions de chacun annoncées, commencèrent les escarmouches. L'Argentine défendait son projet d'arbitrage obligatoire. Le Mexique avoua que les Etats-Unis lui avaient directement proposé un traité d'arbitrage qu'il acceptait, et souhaitait

10 Martí, José, "La Conferencia de Washington, 18 de abril de 1890 ", Nuestra América, -s.l., Biblioteca Ayacucho, ${ }^{\circ} 15,1977$, p. 85. 
inclure certaines exceptions au traité proposé. Le Chili, qui sortait de la Guerre du Pacifique en tant quagresseur, insinua que le projet d'arbitrage, sous couvert de maintien de la paix, était une attaque collective contre lui, et affirma, par conséquent, sa détermination à poursuivre dans la position qu'il s'était fixée. Le Guatemala suivait l'avis de l'Argentine et pensait que "ôter à l'arbitrage son caractère obligatoire équivaudrait à n'avoir rien fait du tout 11 ". Le Pérou annonça qu'il voterait le projet d'arbitrage seulement si le projet " contre la conquête " était voté. La délégation des Etats-Unis montrait des divergences en son sein, entre Henderson le président de la délégation, qui acceptait l'accord, et Trescott le porte parole du gouvernement, qui le rejetait. Finalement, ce fut un problème de procédure qui condamna le projet.

Pour leur part, les républiques latino-américaines préférèrent projeter de discuter entre elles seules de leurs intérêts politiques et économiques communs au lieu de les subordonner au jugement des Nord-américains. Certains délégués latino-américains (les Argentins Quintana et Sáenz Peña et le Guatémaltèque Cruz en particulier) dénoncèrent la politique agressive des Etats-Unis : " Devant le droit international américain (...) il n'existe en Amérique ni grandes ni petites nations : elles sont toutes souveraines et indépendantes de la même manière : elles sont toutes dignes de la même considération et du même respect 12 ". Ainsi, à l'invitation amicale succéda la réserve, la méfiance, puis se fermèrent les possibilités d'entente. Et même si le panaméricanisme ne devint pas effectif à cette époque, la Conférence de Washington marqua un tournant dans les rapports hémisphériques puisqu'elle constitua le point de départ de l'attitude panaméricaniste qui prévaudra pendant le siècle suivant.

\section{Le modèle panaméricain de 1890}

D'inspiration nord-américaine, ce modèle n'est lié ni aux idées et aux efforts de Bolívar ni à l'une des tentatives hispano-américaines d'union du XIXe siècle, quelles qu'elles soient. Ce qui caractérise le plus spécifiquement les relations panaméricaines est une asymétrie des pouvoirs. Le gouvernement des Etats-Unis fut le premier à reconnaître les républiques hispano-américaines indépendantes, attendant avidement l'ouverture d'un nouveau marché de dimension continentale un peu trop exclusivement tourné, selon lui, vers la Grande-Bretagne et la France. Dès 1823, c'est la Doctrine Monroe qui est formulée, considérant toute tentative de reconquête d'un quelconque territoire du continent américain comme une menace contre les intérêts des Etats-Unis autant

11 Ibid., p. 90.

12 Ibid., p. 86. 
que contre ceux d'un autre pays américain. Cette doctrine énonça donc clairement le fait que les Etats-Unis étaient contre tout rétablissement de colonies européennes en Amérique latine ou toute création de protectorats ou encore toute restauration monarchique, et qu'ils étaient prêts à défendre leur conception par les armes. La position des Etats-Unis révélait un isolationnisme hémisphérique qui avait pour but d'assurer leurs intérêts économiques, grâce à un maintien des pays latinoaméricains en dehors des relations mondiales. En 1845, ce fut la doctrine du Destin Manifeste qui interpréta d'une manière nouvelle le message de Monroe pour en faire une couverture idéologique de l'expansionnisme nord-américain.

Le modèle panaméricain de 1890 définissait un système de relations internationales non unioniste, car il existait une méfiance et un refus de ce système de la part des gouvernements latino-américains qui sentaient leur souveraineté menacée. Le postulat sur lequel se fondait cette doctrine était l'existence d'une seule Amérique, un postulat qui différait de celui qu'avaient formulé Bilbaol3 ou Martí, qui en comptaient deux. Cet expansionnisme politique dissimulait un expansionnisme commercial, qui se manifestera, surtout au XXe siècle, en Amérique centrale et dans les Caraïbes, où se succéderont les interventions armées, les pressions politiques et économiques, les gouvernements marionnettes, les assassinats. Les Etats-Unis recherchaient avant tout à travers le panaméricanisme une possibilité d'expansion utilitaire grâce à une politique d'absorption économique.

Le XXe siècle connaîtra un recul du processus d'union en Amérique latine. Pour Mario Amadeo, cette période montre un système (panaméricain) qui se présente comme un groupement régional mais qui n'est qu'une forme sans contenu. Amadeo ne voit dans le chemin qui va de la $1^{\circ}$ Conférence Internationale Américaine de Washington (1889-90) à la $9^{\circ}$ Conférence Interaméricaine de Bogota (1948) qu'une acceptation progressive de la part du continent latino-américain de la tutelle des Etats-Unis, puisque ces derniers réussirent à imposer leur domination sous des perspectives structurelles.

En réalité, le panaméricanisme était un comportement politique, pas une idéologie unioniste, et son objectif était une subordination, pas une union. S'il a effectivement pu jouer un rôle positif dans le maintien et le renforcement du latino-américanisme, c'est que la volonté étasunienne de pénétration économique et politique qu'il véhiculait a poussé les républiques latino-américaines à redoubler de vigilance à travers la recherche d'une unité. A l'issue de la Conférence de Washington, les circonstances imposaient pourtant l'union, car les relations hémisphériques devenaient déséquilibrées en termes de pouvoir. C'était

13 Bilbao, Francisco, Iniciativa de la América: Idea de un Congreso Federal de las Repuiblicas, Paris, 22 juin 1856, México, UNAM, Latinoamérica n 3, p. 12. 
une époque où l'idée de "patrie américaine ", telle que l'entendait José Martí, était tout le contraire d'une utopie. Les circonstances d'un danger interventionniste étaient tangibles, et c'est ce qui amena le Cubain à déclarer qu'il était temps que l'Amérique gagne "sa seconde indépendance ", et ce qui lui inspira un américanisme anti-impérialiste.

C'est d'ailleurs souvent face au panaméricanisme que s'est affirmée la notion d'américanité "du Sud ", l'expression d'une " continentalité " latino-américaine formulée et reformulée par de nombreux hommes politiques et intellectuels latino-américains qui représentent l'esprit " continentaliste " de l'Amérique latine. Mais c'est aussi très souvent face au manque d'action coordonnée entre les jeunes républiques hispanoaméricaines que certains intellectuels et hommes politiques se sont passionnés pour la cause unioniste. Leurs postulats et leurs projets ont contribué à créer un "mythe de l'unité ", l'union étant envisagée comme objectif à atteindre en vue de cet idéal unitaire. Le XIXe siècle a vu naître les théories unionistes sous la plume des personnalités latino-américaines les plus éminentes. Considérés tantôt comme utopistes, tantôt comme visionnaires, ils ont donné un sens global, continental, à l'idée de "lo latinoamericano " et ont progressivement construit un corps théorique relatif à l'unité et à l'union latino-américaines.

\section{Bibliographie}

Amadeo, Mario, Por una convivencia internacional: bases para una comunidad hispánica de naciones, Madrid, Ed. Cultura Hispánica, 1956, 229 p.

Arosemena, Justo, "Estudio sobre la Idea de una Liga Americana", Tareas, Panamá, $\mathbf{n}^{\circ} 26$, jun-nov. 1973, p.5-105.

Bilbao, Francisco, Iniciativa de la América: Idea de un Congreso Federal de las Repúblicas, México, UNAM, 1978, 26 p. (Latinoamérica, Cuadernos de Cultura latinoamericana, $n^{\circ} 3$ ).

Conferencias internacionales americanas, México, Secretaría de Relaciones Exteriores, 1990, 3 vol.

"Convocatoria del Congreso de Panamá " (firmada por el Libertador en Lima el 7 de diciembre de 1824, dos días antes de la batalla de Ayacucho), dans : Simón Bolivar. Doctrina del Libertador, pról. Augusto Mijares, comp., notas y cronol. Manuel Pérez Vila, Caracas, Biblioteca Ayacucho, 1979, p. 177-180.

Galich, Manuel, "A 150 años del Congreso de Panamá : bolivarismo y panamericanismo ", Casa de las Américas, La Habana, n 96, mayo-junio, 1976, p. 4-17. 
González Prieto, Alejandro, "Antecedentes históricos del ideal latinoamericano ", Pensamiento político, México, $n^{\circ} 63$, XVII, julio de 1974, p. 311-326.

Heredia, Edmundo A., "Primeras misiones integracionistas latinoamericanas (1810-26)", Anuario de Estudios Hispanoamericanos, Sevilla, $\mathbf{n}^{\circ} 2, \mathrm{~L}, 1993$, p. 187-242.

Lastarria, José Victorino [et al.], Colección de ensayos y documentos relativos a la unión y confederación de los pueblos hispanoamericanos, Santiago de Chile, Imprenta Chilena, 1862.

Lerman Alperstein, Aída, "Ideas y proyectos de la integración latinoamericana : siglo XIX y primera mitad del siglo XX", Mundo Nuevo, Caracas, IAEAL, n 65-66, XVII, julio-dic., 1994, p. 307-324.

Martí, José, "La Conferencia de Washington, 18 de abril de 1890 ", Nuestra América, -s.l., Biblioteca Ayacucho, nº 15, 1977.

Martínez, Ricardo A., De Bolivar a Dulles. El panamericanismo, doctrina y práctica imperialista, México, Ed. América Nueva, 1959, 229 p. (Col. Autores contemporáneos, $\left.\mathbf{n}^{\circ} 17\right)$.

Orrego Vicuña, Eugenio, Ensayos. I. Los problemas de la unificación americana (siglos XVIII y XIX), Santiago de Chile, Universidad de Chile, 1947, p. 153-203.

Porras Barrenechea, Raúl (recop. y pról.), El Congreso de Panamá, Lima, imp. "La Opinión Nacional ", 1930, CI-500 p. (Archivo diplomático del Perú, $\left.n^{\circ} 1\right)$.

Torres Caicedo, José María, Unión Latino-Americana: pensamiento de Bolivar para formar una liga americana, su origen y sus desarrollos, Paris, Rosa y Bouret, 1865, 385 p.

Townsend Ezcurra, Andrés, "La nación de repúblicas: proyecto latinoamericano de Bolívar", Integración latinoamericana, Buenos Aires, INTAL, $\mathbf{n}^{\circ}$ 146-147, XIV, junio-julio, 1989, p. 52-67.

Vargas, Francisco Alejandro, El Congreso anfictiónico de Panamá y su proyección hacia el futuro de la América, Caracas, Oficina Técnica M. D., 1976. 
RÉSUMÉ- Le modèle bolivarien globalisant trouve son origine dans la certitude qu'une sorte de géopolitique de blocs était nécessaire au XIXe siècle sur le continent américain. A partir du Congrès Amphictyonique de Panama (1826) tous les grands projets unitaires latino-américains furent pensés dans cette perspective : fédérations, confédérations, alliances permanentes. On pouvait l'observer dans la tenue d'assemblées, de congrès, de conférences, de traités d'amitié qui, avec de grandes déficiences, des limites et peu de résultats, rendaient manifeste l'existence d'une communauté et d'un mouvement insistant vers l'union.

RESUMEN- El modelo globalizador bolivariano se basa en la certidumbre de que una geopolítica de bloques era necesaria en el siglo XIX en el continente americano. A partir del Congreso Anfictiónico de Panamá (1826) todos los grandes proyectos unitarios latinoamericanos se concibieron según esta óptica: federaciones, confederaciones, alianzas permanentes. Se podía notar en la realización de asambleas, congresos, conferencias, tratados de amistad, los cuales, con grandes deficiencias, limitaciones, y pocos resultados, revelaban la existencia de una comunidad y un movimiento insistente hacia la unión.

ABSTRACT - The Bolivarian globalizing model has its origin in the strong belief that a kind of geopolitics based on blocks was necessary in the $19^{\text {th }}$ century in the American continent. After Panama's Amphictyonic Congress (1826), all the great Latin American unitary projects were contemplated through this perspective: federations, confederations, permanent alliances. This can be observed in the way assemblies, congresses, conferences and friendship treaties were held, which, with great lacks, limits and few results, made obvious the existence of a community and of a movement aiming towards union.

MOTS-CLES : Unité latino-américaine, XIXe siècle, Traités, Alliances, Bolivar. 